\title{
Postsurgical Stage IV Hepatoblastoma
}

National Cancer Institute

\section{Source}

National Cancer Institute. Postsurgical Stage IV Hepatoblastoma. NCI Thesaurus. Code C7143.

Distant metastases, regardless of the extent of liver involvement. (from PDQ 2004) 